\title{
A case of cerebral malaria: are we alert to detect?
}

\author{
GJ Pratheepan ${ }^{1}$, CK Bodinayake ${ }^{2}$ \\ ${ }^{1}$ Registrar in Medicine, ${ }^{2}$ Consultant Physician,University Medical Unit, Teaching Hospital, Karapitiya, \\ Galle.
}

\section{Introduction}

Malaria is an important treatable cause of fever. Cerebral malaria caused by Plasmodium falciparum causes multisystem involvement leading to death. Though malaria was a common illness in Sri Lanka years back, the incidence is now very low. Total of 196 cases of malaria and 8 cases of cerebral malaria were reported in $2007^{1}$.

We report a case of cerebral malaria from Galle with many of its complications.

\section{Case report}

A sixty year old patient from Panangala was transferred to Teaching Hospital Karapitiya from Udugama hospital with a history of intermittent high fever, dysuria, abdominal pain and confusion for three days. He had anorexia, malaise and myalgia. He had no past history of malaria and has not traveled to a malaria endemic area.

On examination, he was drowsy. Temperature was $102^{\circ} \mathrm{F}$. Mild icterus and conjunctival suffusion were present. There was no neck stiffness. His pulse was $100 \mathrm{bpm}$ and blood pressure was $110 / 70 \mathrm{mmHg}$. There were bilateral basal crepitations in the lungs. Abdominal examination revealed $2 \mathrm{~cm}$ hepatomegaly and suprapubic tenderness. Spleen was not palpable.

During ward stay he had rigors with drenching sweats. He became increasingly drowsy, restless and developed abnormal rapid breathing.
Patient received IV Broad spectrum antibiotics as for septicaemia as he had neutrophil leucocytosis and moderately field full pus cells in urine. His blood glucose remained persistently below $2.2 \mathrm{mmol} / \mathrm{L}$.

Fever with rigors, confusion, hypoglycaemia and absence of neck stiffness led to the clinical suspicion of cerebral malaria. Thick and thin blood films showed Plasmodium falciparum in its ring stages.

\section{Investigations}

He had a white cell count of $17,700 / \mathrm{mm}^{3}$ with $82 \%$ neutrophils, $13 \%$ lymphocytes, $2 \%$ eosinophils and 3\% monocytes. His platelet count was $26,000 / \mathrm{mm}^{3}$ and $\mathrm{Hb}$ was $12.7 \mathrm{~g} / \mathrm{dL}$. Blood picture showed normocytic normochromic red cells with neutrophil leukocytosis and thrombocytopenia. UFR revealed moderately field full pus cells and red cells.

His FBS was $1.5 \mathrm{mmol} / \mathrm{L}$, RBS was $2.2 \mathrm{mmol} / \mathrm{L}$, blood urea was $65 \mathrm{mg} / \mathrm{dL}$ and serum creatinine was $2.0 \mathrm{mmol} / \mathrm{L}$. He had SGOT of $147 \mathrm{U} / \mathrm{L}$, SGPT of $78 \mathrm{U} / \mathrm{L}$, and INR of 1.6

His ECG was normal. Arterial blood gas analysis showed $\mathrm{PH}$ of 7.386, $\mathrm{PaCO}_{2}$ of 24.4 $\mathrm{mmHg}, \mathrm{PaO}_{2}$ of $88.5 \mathrm{mmHg}, \mathrm{HCO}_{3}{ }^{-}$of 17.7 $\mathrm{mmol} / \mathrm{L}$ and $\mathrm{O}_{2}$ saturation of $96.8 \%$.

Abdominal ultrasound scan revealed bilateral Staghorn calculi. There was no renal parenchymal disease or hydronephrosis. Mild 
hepatomegaly was present with no splenomegaly.

CT brain and Chest X-ray were normal.

Patient subsequently developed diffuse alveolar shadowing consistent with ARDS.

\section{Blood film: Plasmodium falciparum ring stages present.}

\section{Discussion}

World wide, 300 - 450 million people develop malaria, resulting in one million deaths annually 2. Plasmodium falciparum cause severe illness leading to complications as cerebral malaria, severe anaemia, renal failure, pulmonary oedema, hypoglycaemia, circulatory collapse, hyperpyrexia, acute renal failure, acute hepatopathy with centrilobular necrosis and jaundice, hypoglycemia, cardiac dysrhythmias, gastrointestinal syndromes, acidosis and water and electrolyte disturbances.

This case illustrates cerebral malaria presenting as an acute febrile illness with severe hypoglycaemia, thrombocytopenia, hepatic and renal impairment, acidosis and developing ARDS as a life threatening complication.

Hypoglycaemia in cerebral malaria is well known and contributes to nervous system dysfunction and residual neurological effects in the survivors. Hypoglycaemia is associated with hyperlactataemia sharing the same pathophysiological mechanisms ${ }^{3}$. These include the increased metabolic demands of febrile illness, obligatory demands of the parasite which use glucose as the major fuel, failure of hepatic gluconeogenesis and glycogenolysis. The hepatic stores of glycogen last approximately two days in adults and 12 hours in children ${ }^{3}$. All these factors contribute to develop hypoglycaemia in $20-30 \%$ children and $10 \%$ of adults with severe malaria ${ }^{3}$. Treatment with Quinine which stimulates pancreatic beta cells is balanced by a reduced sensitivity to insulin. This tends to occur after the first 24 hours of treatment whereas malaria related hypoglycaemia is often present before the treatment is initiated.

The patient received IV quinine and IV $10 \%$ glucose infusions and additional supportive care. Current malaria treatment guidelines include artimensin compounds as first line therapy as they are highly effective and rapidly schzonticidal. A large multi-center randomized placebo-control trial carried out in South-east Asia has reported $35 \%$ reduction in the mortality with parenteral (IV) artesunate as compared to IV quinine. Artesunate use was associated with very few adverse events in this study ${ }^{4}$. In Sri Lanka the current malaria guidelines recommend oral Artemensin based combination therapy (ACT) for Falciparum infections in adults and children weighing $>5 \mathrm{~kg}$ who can receive oral therapy ${ }^{5}$.

As the patient developed respiratory distress he was managed in the ICU and ventilated for 72 hours. He steadily improved and was discharged after 14 days.

This case report highlights the need for clinician's awareness to consider cerebral malaria even from nonendemic areas if the symptoms are suggestive. It also illustrates the many possible life threatening complications that need to be detected and managed for a positive outcome.

\section{References}

1. Anti Malaria campaign Sri Lanka (Unpublished data).

2. World malaria report 2005. rbm.who.int/wmr $2005 /$ html/a1_l.htm

3. Gordon Cook, Alimuddin Zumla Manson's tropical diseases ( $21^{\text {st }}$ edition).

4. Dondorp A, Nosten F, Stepniewska K, Day N, White $\mathrm{N}$; Artesunate versus quinine for treatment of severe falciparum malaria: Lancet 2005; 366: 717-25. 